International Journal of Trend in Scientific Research and Development (IJTSRD)

Volume: 3 | Issue: 3 | Mar-Apr 2019 Available Online: www.ijtsrd.com e-ISSN: 2456 - 6470

\title{
Analysis of Energy in Wireless Sensor Networks: An Assessment
}

\author{
Anupam Jain'1, Prof. Madhuvan Dixit ${ }^{2}$ \\ 1PG Scholar, ${ }^{2}$ Assistant Professor \\ 1,2Department of CSE, MIT, Bhopal, Madhya Pradesh, India
}

\begin{abstract}
How to cite this paper: Anupam Jain | Prof. Madhuvan Dixit "Analysis of Energy in Wireless Sensor Networks: An Assessment" Published in International Journal of Trend in Scientific Research and Development (ijtsrd), ISSN: 24566470, Volume-3 | Issue-3, April 2019, pp.267-271, URL: http://www.ijtsrd.co $\mathrm{m} /$ papers/ijtsrd 227 89.pdf

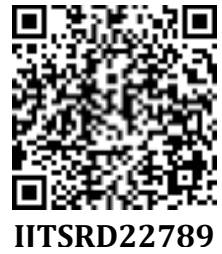

Copyright (C) 2019 by author(s) and International Journal of Trend in Scientific Research and Development Journal. This is an Open Access article distributed under the terms of the Creative Commons

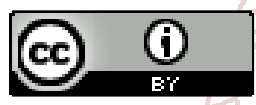
Attribution License (CC BY 4.0) (http://creativecommons.org/licenses/ by/4.0)

\section{INTRODUCTION}

Wireless Sensor Networks(WSN's) is a network consisting of numerous sensor nodes. A sensor node(SN) is a multifunctional, low-power and low-cost tiny size device. SNs are randomly deployed over a zone to measure various phenomena like humidity, moisture, vibrations and many more. By enhancing the capabilities of sensor nodes, realization of WSNs based on the collaborative effort of sensor nodes is easy[1]. SN has four basic components Sensing unit, Processing unit, Transceiver unit and Power unit are shown in Figure 1.SNsare broadly classified as normal nodes, advanced nodes and super nodes. Apparently, the normal nodes have the least energy level, the advanced nodes have more energy than the normal nodes and the super nodes have the highest level of energy[2].The major advantages of SNs in comparison to the normal nodes are their ability to operate in harsh environment in which contemporary monitoring is risky and sometime not feasible. SN's are established randomly in field of interest by uncontrolled means like dropped by a helicopter etc. The general architecture of WSN's is shown in Figure 2. SNs collect data and transmit to the base station or sink node[3]. Therefore data collection can be single hop or multi-hop. Sink node is an intermediate node which receives the data from the sensor field and transmits over the internet. In WSN's, SNs are randomly setup in a geographical region. This region is divided into sub-regions called clusters. In each cluster, one of the node is selected as cluster head $(\mathrm{CH})$ and the remaining nodes are cluster members[4]. $\mathrm{CH}$ is chosen on the basis of weighted probability. The node having highest weight probability has more chance to become a $\mathrm{CH}$. Weight probability is the ratio of residual energy of node and average energy of wireless sensor network.

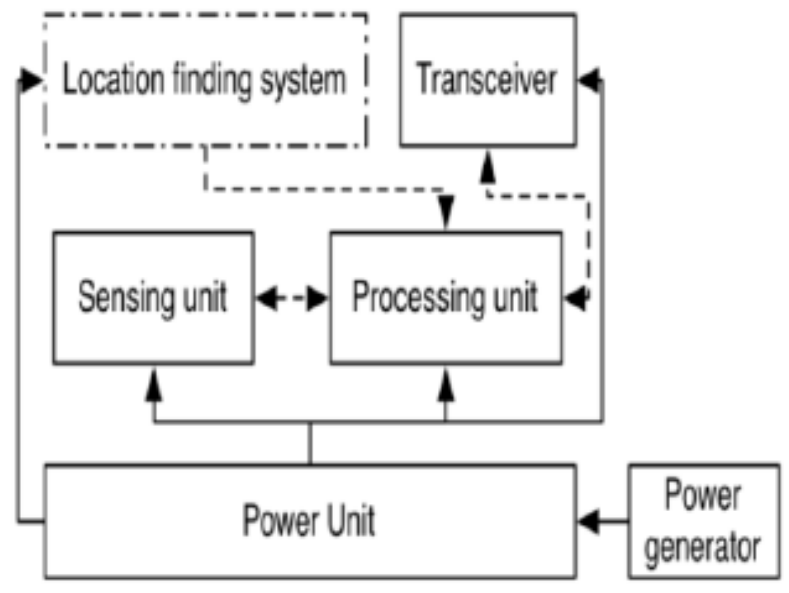

Figure 1: Components of sensor node 


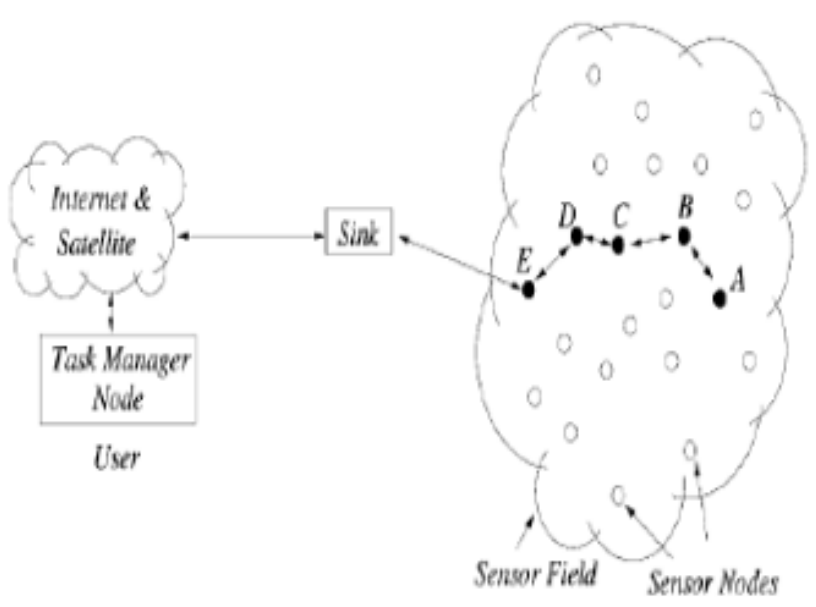

Figure 2: WSN's architecture

The rest of the paper is structured as follows. Sections II presents the related work on various aspects of energy management in WSNs. Discussion of various energy consumption protocols is carried out in section III and Section IV concludes the paper.

\section{RELATED WORK}

Internet of thing (IoT) is not only a promising research topic but also a blooming industrial trend. Although the basicidea is to bring things or objects into the Internet, there are various approaches because an IoT system is highly application oriented. In addition to the design of a customized hardware for range extension, a new synchronization scheme and a burst transmission feature are also presented to boost the network capacity and reduce the energy waste. As a result, the proposed platform can fulfill the high throughput requirement for high-rate applications and the requirement of long battery life for lowrate applications at the same time. We have developed a test bed in our campus to validate the proposed system.(YawWen Kuo, Cho-Long Li, Jheng-Han Jhang and Sam Lin; 2018)

In wireless sensors networks, the sensor nodes are densely deployed. Owing to this excessive deployment of sensor nodes, each target is covered by multiple sensors at a time. To prolong the network lifetime, the authors can schedule the sensor activity in such a way that only a subset of sensor nodes, called cover set, is sufficient enough to cover all the targets. In this study, they propose an energy-efficient scheduling algorithm based on learning automata for target coverage problem. The learning automata-based technique helps a sensor node to select its appropriate state (either active or sleep). To prove the effectiveness of their proposed scheduling method, they conduct a detailed set of simulations and compare the performance of their algorithm with the existing algorithms.(Manju, Satish Chand, Bijender Kumar; 2018)

This work presents a combined energy-efficient medium access control (MAC) and routing protocol for large-scale wireless sensor networks that aims to minimize energy consumption and prolong the network lifetime. The proposed communication framework employs the following measures to enhance the network energy efficiency. Firstly, it provides an adaptive intra-cluster schedule to arbitrate media access of sensor nodes within a cluster, minimizing idle listening on sensor nodes, leading to improved energy performance. Secondly, it proposes an on-demand source cross-layer routing protocol ensuring selection of best routes based on energy level and channel quality indicator for the multi-hop inter-cluster data transmission. Lastly, an unequal cluster size technique based on cluster head residual energy and distance away from the base station is utilized. This technique balances the energy among clusters and avoids early network partitioning. This work further presents the analytical performance model for energy consumption and delay of the proposed communication framework. The performance measures used for evaluation are energy consumption, delay, and network lifetime. The results indicate that combining routing and MAC schemes conserves energy better than utilizing MAC scheme alone.(Maria Sefuba, Tom Walingo; 2018)

3-axis accelerometer is a widely adopted device for applications such as fall detection of the elders, kinematic analysis, building/bridge/landslide monitoring, and so on. To retrieve the sensing data in real time, one may use a wireless module to forward those data to a gateway or server. For outdoor applications, Zigbee/IEEE 802.15.4 technology is often the candidate because it is cost effective and easy to be purchased in the market. However, transmission range, energy consumption and network capacity are key factors in designing an outdoor system. (Yaw-Wen Kuo and Cho-Long Li; 2017).

Time-synchronized channel hopping (TSCH) is currently the most efficient solution for collision-free, interference avoiding communications in ad hoc wireless networks, such as wireless sensor networks, vehicular networks, and networks of robots or drones. However, all variants of TSCH require some form of centralized coordination to maintain the time-frequency slotting mechanism. This leads to slow convergence to steady state and moderate time-frequency slot utilization, especially under node churn or mobility. We propose decentralized timesynchronized channel swapping (DT-SCS), a novel protocol for medium access control (MAC) in ad hoc wireless networks. Under the proposed protocol, nodes first converge to synchronous beacon packet transmissions across all available channels at the physical layer, with balanced number of nodes in each channel. (George Smart, Nikos Deligiannis, Rosario Surace, Valeria Loscri, Giancarlo Fortino and Yiannis Andreopoulos; 2016).

We have witnessed the Fixed Internet emerging with virtually every computer being connected today; we are currently witnessing the emergence of the Mobile Internet with the exponential explosion of smart phones, tablets and net-books. However, both will be dwarfed by the anticipated emergence of the Internet of Things (IoT), in which everyday objects are able to connect to the Internet, tweet or be queried. Whilst the impact onto economies and societies around the world is undisputed, the technologies facilitating such a ubiquitous connectivity have struggled so far and only recently commenced to take shape. To this end, this paper introduces in a timely manner the cornerstones of a technically and commercially viable IoT which includes a detailed discussion on the particular standard of choice at each protocol layer. This stack is shown to meet the important criteria of power-efficiency, reliability and Internet connectivity. Industrial applications have been the early adopters of this stack, which has become the de-facto standard, thereby bootstraping early IoT developments. 
Corroborated throughout this paper and by emerging industry alliances, we believe that a standardized approach, using latest developments in the IEEE 802.15.4 and IETF working groups, is the only way forward. (Maria Rita Palattella, Nicola Accettura, Xavier Vilajosana, Thomas Watteyne, Luigi Alfredo Grieco, Gennaro Boggia and Mischa Dohler; 2016)

The main concern in Wireless Sensor Networks is how to handle with their limited energy resources. The performance of Wireless Sensor Networks strongly depends on their lifetime. As a result, Dynamic Power Management approaches with the purpose of reduction of energy consumption in sensor nodes, after deployment and designing of the network. Recently, there have been a strong interest to use intelligent tools especially Neural Networks in energy efficient approaches of Wireless Sensor Networks, due to their simple parallel distributed computation, distributed storage, data robustness, auto classification of sensor nodes and sensor reading. This paper presents a new centralized adaptive Energy Based Clustering protocol through the application of Self organizing map neural networks (called EBC-S) which can cluster sensor nodes, based on multi parameters; energy level and coordinates of sensor nodes. We applied some maximum energy nodes as weights of SOM map units; so that the nodes with higher energy attract the nearest nodes with lower energy levels. Therefore, formed clusters may not necessarily contain adjacent nodes. The new algorithm enables us to form energy balanced clusters and equally distribute energy consumption. Simulation results and comparison with previous protocols( LEACH and LEA2C) prove that our new algorithm is able to extend the lifetime of the network.(Mohammad Ali Azimi kashani and Hassan Ziafat; 2016)

The increasing complexity of Wireless Sensor Networks (WSNs) is leading towards the deployment of complex networked systems and the optimal design of WSNs can be a very difficult task because several constraints and requirements must be considered, among all the power consumption. This paper proposes a novel fuzzy logic based mechanism that according to the battery level and to the ratio of Throughput to Workload determines the sleeping time of sensor devices in a Wireless Sensor Network for environmental monitoring based on the IEEE 802.15.4 protocol. The main aim here is to find an effective solution that achieves the target while avoiding complex and computationally expensive solutions, which would not be appropriate for the problem at hand and would impair the practical applicability of the approach in real scenarios. The results of several real test-bed scenarios show that the proposed system outperforms other solutions, significantly reducing the whole power consumption while maintaining good performance in terms of the ratio of throughput to workload. An implementation on off-the-shelf devices proves that the proposed controller does not require powerful hardware and can be easily implemented on a low-cost device, thus paving the way for extensive usage in practice.(Giovanni Pau, "Power Consumption Reduction for Wireless Sensor Networks Using A Fuzzy Approach", International Journal of Engineering and Technology Innovation, vol. 6, no. 1, 2016)
Energy management in Wireless Sensor Networks (WSNs) is of paramount importance for the remotely deployed energy stringent sensor nodes. These nodes are typically powered by attached batteries. Several battery-driven energy conservation schemes are proposed to ensure energy efficient network operation. The constraints associated to the limited battery capacity shifted the research trend towards finding alternate sources by harvesting ambient energy. This survey presents a high level taxonomy of energy management in WSNs. We analyze different battery-driven energy consumption based schemes and energy harvesting based energy provision schemes. We also highlight the recent breakthrough of wireless energy transference to a sensor node as an alternative to typical batteries. We recommend take into account recent energy provisioning advancements in parallel with the traditional energy conservation approaches for a sensor network while designing energy efficient schemes.(Junaid Ahmed Khan, Hassaan Khaliq Qureshi and Adnan Iqbal, "Energy management in Wireless Sensor Networks: A survey", https://hal.archives-ouvertes.fr/hal-01283728, 2015)

\section{DISCUSSION}

Various protocols discussed in section II are analyzed and their outcomes/methodology are presented in tabular form as shown in table I. WSN approaches discussed in section-II have been categorized into three main catagories: datacentric routing protocol, hierarchical protocols and aggregation based protocols. In the field of WSN's, LEACH protocol solves the problem of energy consumption due rotation of cluster heads in setup phase of cluster formation [4]. In LEACH, data gathering problem developed does not reach an optimal solution. PEGASIS protocol was developed for solving data gathering problem. The key point of PEGASIS is to make chain to nearest sensor node so that each node can receive and transmit data to closest SN[5]. PEGASIS gives better performance in comparison to LEACH. In both LEACH and PEGASIS network simulators are used to measure the efficiency of WSN's. LEACH doesn't have the data-centric routing capability. A new protocol called REEP was developed for reliable and energy efficient data-centric routing[6]. In data-centric routing, the BS sends queries to certain regions and waits for data from deployed sensors, hence data is collected through queries. EEHCA was developed to overcome the problem of load balancing. EEHCA is a hierarchical and data aggregation based WSN protocol. EEHCA increased the WSN's lifetime with the help of backup cluster heads. In EEHCA if the primary $\mathrm{CH}$ is depleted of all its energy then secondary $\mathrm{CH}$ manages the load. EEHC was developed for energy efficiency maximization on the basis of weighted election probability of their residual energy.To fulfil the objective of energy efficient and load balancing scheme, a approach called S-EECP and M-EECP was developed. In SEECP the single hop communication is established inside the clusters between non-CHs and $\mathrm{CHs}$ whereas multi-hop communication is established between $\mathrm{CHs}$ and BS by MEECP[10]. The latest protocol for achieving the mobility and decreasing the delay in transmission of data is VELCT. VELCT construct the DCT based on cluster head location which helps in reducing the end to end delay of network. 
International Journal of Trend in Scientific Research and Development (IJTSRD) @ www.ijtsrd.com eISSN: 2456-6470

Table 1: Comparative analysis of different algorithms and protocol

\begin{tabular}{|c|c|c|c|c|c|}
\hline Technique & Objective & $\begin{array}{l}\text { Simulator } \\
\text { Used }\end{array}$ & $\begin{array}{l}\text { Data- } \\
\text { centric }\end{array}$ & $\begin{array}{l}\text { Hierarchi } \\
\text { cal }\end{array}$ & $\begin{array}{l}\text { Data } \\
\text { aggregatio } \\
\mathbf{n}\end{array}$ \\
\hline LEACH & $\begin{array}{l}\text { Increase } \\
\text { lifetime and } \\
\text { quality of network } \\
\text { latency Decrease }\end{array}$ & $\begin{array}{l}\text { Network } \\
\text { simulator(NS) }\end{array}$ & & $\checkmark$ & $\checkmark$ \\
\hline PEGASIS & $\begin{array}{l}\text { Removed } \\
\text { ata gathering } \\
\text { problem }\end{array}$ & NS-2 & $\checkmark$ & $\checkmark$ & $\checkmark$ \\
\hline RFEP & $\begin{array}{l}\text { Increase } \\
\text { reliability of } \\
\text { routing } \\
\text { of WSN's } \\
\text { Fatult- } \\
\text { tolerance }\end{array}$ & Matlab 7.4 & $\checkmark$ & & $\checkmark$ \\
\hline EEHCA & $\begin{array}{l}\text { Balancing } \\
\text { energy load } \\
\text { lifetime of WSN's }\end{array}$ & Matlab & & $\checkmark$ & $\checkmark$ \\
\hline EEHC & $\begin{array}{l}\text { Energy } \\
\text { efficient }\end{array}$ & $\begin{array}{l}\text { Network } \\
\text { simulator(NS) }\end{array}$ & & $\checkmark$ & $\checkmark$ \\
\hline MRA-AHP & $\begin{array}{l}\text { Load } \\
\text { balancing } \\
\text { Energy } \\
\text { efficient }\end{array}$ & NS-2 & & $\checkmark$ & \\
\hline $\begin{array}{l}\text { S-EECP } \\
\text { \&-M-EECP }\end{array}$ & $\begin{array}{l}\text { Energy } \\
\text { efficient } \\
\text { balancing }\end{array}$ & NS-2 & & $\checkmark$ & \\
\hline VEICT' & - Mobility & NS-2 & & & \\
\hline
\end{tabular}

Further in depth analyzation the effect of different parameter such as load balancing, reliability, ideal listening and latency in data transmission. The first factor, load balancing is the distribution of energy load throughout the WSN's for maximizing the network lifetime. The primary objective of protocol listed in Table 2 is balancing the energy load and providing a reliable communication between SNs[9]. Reliability means protocol is working according to their functionality or specification without any failure. Ideal-listening occurs when a node is not receiving any message but still in an active state for incoming messages, hence the energy is consumed. In order to reduce the energy consumption due to idle-listening in WSN a protocol called Sparse Topology and Energy Management (STEM) has been developed. STEM efficiently wakes up nodes from a sleep state to active state when there is any message to receive. However the nodes don't suffer from idle-listening. Latency means delay in packet transmission; hence more energy is consumed if packet takes a long route. REEP, S-EECP, M-EECP and VLECT protocol suffer with problem of ideal-listening.

Table 2: Effect of various techniques over selected parameters

\begin{tabular}{|l|l|l|l|l|}
\hline $\begin{array}{l}\text { FactorsProtoc } \\
\text { ols }\end{array}$ & Load balancing & Reliability & Ideal-listening & $\begin{array}{l}\text { Decrease } \\
\text { Latency }\end{array}$ \\
\hline LEACH & $\checkmark$ & $\checkmark$ & & $\checkmark$ \\
\hline REEP & $\checkmark$ & $\checkmark$ & $\checkmark$ & $\checkmark$ \\
\hline MRA-AHP & $\checkmark$ & $\checkmark$ & & $\checkmark$ \\
\hline $\begin{array}{l}\text { S-EECP\& } \\
\text { M-EECP }\end{array}$ & $\checkmark$ & $\checkmark$ & $\checkmark$ & $\checkmark$ \\
\hline VLECT & $\checkmark$ & $\checkmark$ & $\checkmark$ & $\checkmark$ \\
\hline
\end{tabular}


On analyzation of WSN protocols we have found some research gapes. In EEHCA the data transmission capability and inter cluster communication can be improved. The REEP protocol has been designed for static nodes only so it can also be design for mobile node which will helpful for tracking moving devices. In REEP lots of energy is waste when the node in active mode so it's also design for sleep or active mode operation for saving energy and lifetime of WSN is increases.

Another interesting issue in energy consumption protocols is the selection of cluster head among available sensor nodes. A cluster head perform data aggregation and transmition to BS. Many researchers have been done in WSNs to build optimized clusters with efficient and reliable data collection techniques, but still there are research gaps in cluster formation and $\mathrm{CH}$ selection. Most of the protocol assumes that sink node is stationary. However there are some situations where the sink node is mobile like a battlefield where the information of enemy and battleships current location are frequently transmitted to the control rooms hence the sensors energy drains rapidly. So new protocols are needed for handling the changes in topology and mobility overhead.

\section{CONCLUSION AND FUTURE WORKS}

At present, WSNs is an emerging research area. In this paper we have summarized the WSNs protocols and proposed a comparative study based on the data-centric routing, hierarchical and aggregation based protocol. Furthermore, the protocol is classified based on the energy consumption factors like latency, load balancing and energy efficiency. Although many of WSN protocol look promising but still there are many challenges that needs to be solved in WSNs. We highlight those challenges and pointing out future research in the direction to solve energy consumption problem to be solved in WSNs. We highlight those challenges and pointing out future research in the direction to solve energy consumption problem.

\section{REFERENCES}

[1] Yaw-Wen Kuo, Cho-Long Li, Jheng-Han Jhang and Sam Lin, "Design of a wireless sensor network based IoT platform for wide area and heterogeneous applications", IEEE Sensors Journal, 2018.

[2] Manju, Satish Chand, Bijender Kumar, "Target coverage heuristic based on learning automata in wireless sensor networks", IET Wirel. Sens. Syst., 2018.

[3] Maria Sefuba, Tom Walingo, "Energy-efficient medium access control and routing protocol for multihop wireless sensor networks", IET Wireless Sensor Systems, 2018.

[4] Yaw-Wen Kuo and Cho-Long Li, "Design of a long range wireless 3-axis accelerometer module for environmental monitoring", IEEE Journal of WSN, 2017.
[5] Arvind Kumar, Damanpreet Singh, "Importance of Energy in Wireless Sensor Networks: A Survey", An International Journal of Engineering Sciences, Vol. 17, 2016.

[6] George Smart, Nikos Deligiannis, Rosario Surace, Valeria Loscri, Giancarlo Fortino and Yiannis Andreopoulos, "Decentralized Time-Synchronized Channel Swapping for Ad Hoc Wireless Networks", IEEE Transaction of Wireless Network, 2016.

[7] Maria Rita Palattella, Nicola Accettura, Xavier Vilajosana, Thomas Watteyne, Luigi Alfredo Grieco, Gennaro Boggia and Mischa Dohler, "Standardized Protocol Stack For The Internet Of (Important) Things", IEEE Conference of IoT, 2016.

[8] Mohammad Ali Azimi kashani and Hassan Ziafat, "A method for Reduction of Energy Consumption in Wireless Sensor Network with using Neural Networks", IEEE Conference of Wireless Sensor Network, 2016.

[9] Giovanni Pau, "Power Consumption Reduction for Wireless Sensor Networks Using A Fuzzy Approach", International Journal of Engineering and Technology Innovation, vol. 6, no. 1, 2016.

[10] Junaid Ahmed Khan, Hassaan Khaliq Qureshi and Adnan Iqbal "Energy management in Wireless Sensor Networks: A survey", https://hal.archivesouvertes.fr/hal-01283728, 2015.

[11] Keshav Sood, Shui Yu and Yong Xiang, "Software Defined Wireless Networking Opportunities and Challenges for Internet of Things: A Review", IEEE internet of things journal, 2015.

[12] Ala Al-Fuqaha, Mohsen Guizani, Mehdi Mohammadi, Mohammed Aledhari and Moussa Ayyash, "Internet of Things: A Survey on Enabling Technologies, Protocols, and Applications", IEEE communication surveys \& tutorials, vol. 17 , no. 4 , fourth quarter 2015 .

[13] Antonio Guerrieri, Giancarlo Fortino and Wilma Russo, "An Evaluation Framework for Buildings-oriented Wireless Sensor Networks", 14th IEEE/ACM International Symposium on Cluster, Cloud and Grid Computing, 2014

[14] B. Molina, C.E. Palau, G. Fortino, A. Guerrieri, C. Savaglio, "Empowering smart cities through interoperable Sensor Network Enablers", IEEE International Conference on Systems, Man, and Cybernetics, 2014

[15] Mihai T. Lazarescu, "Design of a WSN Platform for Long-Term Environmental Monitoring for IoT Applications", IEEE journal on emerging and selected topics in circuits and systems, vol. 3, no. 1, march 2013. 